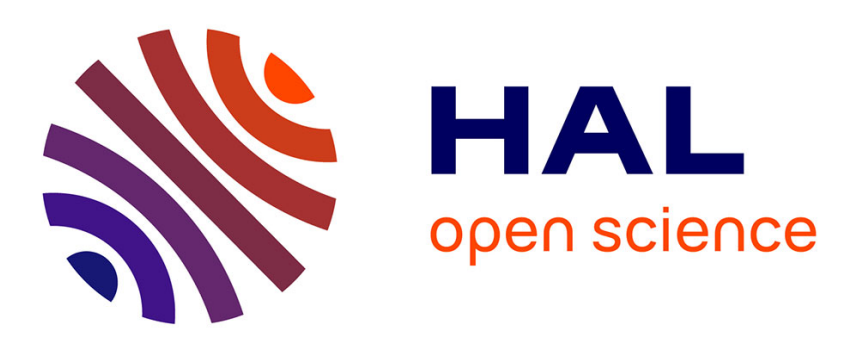

\title{
Theoretical Study of Neutral Dipolar Atom in Water: Structure, Spectroscopy and Formation of an Excitonic State
}

Riccardo Spezia, François-Xavier Coudert, Anne Boutin

\section{To cite this version:}

Riccardo Spezia, François-Xavier Coudert, Anne Boutin. Theoretical Study of Neutral Dipolar Atom in Water: Structure, Spectroscopy and Formation of an Excitonic State. Modern Physics Letters B, 2004, 18 (26/27), pp.1327-1345. 10.1142/S0217984904007827 . hal-00199371

\section{HAL Id: hal-00199371 https://hal.science/hal-00199371}

Submitted on 18 Dec 2007

HAL is a multi-disciplinary open access archive for the deposit and dissemination of scientific research documents, whether they are published or not. The documents may come from teaching and research institutions in France or abroad, or from public or private research centers.
L'archive ouverte pluridisciplinaire HAL, est destinée au dépôt et à la diffusion de documents scientifiques de niveau recherche, publiés ou non, émanant des établissements d'enseignement et de recherche français ou étrangers, des laboratoires publics ou privés. 
Electronic version of an article published as Modern Physics Letters B, Vol. 18, Nos. 26 \& 27 (2004) 1327-1345

(c) World Scientific Publishing Company

\title{
STUDYING NEUTRAL DIPOLAR ATOMS IN WATER VIA ATOMISTIC SIMULATIONS: STRUCTURE, SPECTROSCOPY AND FORMATION OF AN EXCITONIC STATE
}

\author{
Riccardo Spezia *, Francois-Xavier Coudert and Anne Boutin \\ Laboratoire de Chimie Physique, UMR 8000, CNRS, \\ Université Paris-Sud XI, 91405 Orsay Cedex, France
}

Received 29 October 2004

Keywords: Excitonic State; Hydrated Neutral Atoms; Solvated Electron; Condensed Phase Ultrafast Reactions; Molecular Dynamics

\section{Introduction}

Cation reduction in solution is an everyday process having a fundamental role in a broad range of fields, from industrial to biological sciences. For this reason several efforts were done to understand the elementary processes involved. In particular the electron transfer, the crucial step thermodynamically and kinetically regulating redox reactions, was largely investigated during the last decades ${ }^{1}$. Pulse radiolysis of a metal ion in solution offers a powerful method to reduce solvated metal ions and then study by time-resolved spectroscopy the formation of metal atoms and their coalescence ${ }^{2}$. Within this technique a solvated electron is formed, able to reduce the metal ions present in solution.

Different ways can be adopted to generate a solvated electron ${ }^{3}$. Hart and Boag pointed it out in water for the first time in $1962^{4}$, the so-called hydrated electron, its absorption spectrum being a non-structured band with a maximum value at $1.72 \mathrm{eV}$, confirming previous hypothesis of its existence ${ }^{5}$. Then the hydrated electron was largely characterized experimentally, including studies of its stationary spectrum in different conditions ${ }^{6}$. More recently, its formation dynamics became the subject of several studies ${ }^{7}$. Furthermore, in last ten years it was the subject of several theoretical and computational studies ${ }^{8,9}$.

Since the solvated electron is a very strong reducing agent, when produced in a solution it is able to reduce cations. Of course, the reduction process is determined by the chemical nature of the cation. Considering, for simplicity, only monovalent

* present address: Département de Chimie, Ecole Normale Supérieure, UMR 8640, CNRS, 24 rue Lhomond, F-75231 Paris Cedex 05, France; e-mail address: spezia@ens.fr 
cations (similar considerations can be made for a generally charged cation), when a solvated electron is produced in a solution containing a soluble salt of this cation, we have two possible processes

$$
\mathrm{M}^{+}+e_{\mathrm{sol}}^{-} \rightleftharpoons \mathrm{M}^{0}
$$

and

$$
\mathrm{M}^{+}+e_{\mathrm{sol}}^{-} \rightleftharpoons\left(\mathrm{M}^{+}, e_{\mathrm{sol}}^{-}\right)
$$

When the reaction 1 is favorized, then the atoms dimerize or associate with excess ions

$$
\mathrm{M}^{0}+\mathrm{M}^{+} \rightleftharpoons \mathrm{M}_{2}^{+}
$$

and, by a multistep process, these species progressively coalesce into clusters. The most studied reduction reaction assisted by a solvated electron is probably that of the silver cation. It was in fact the first ion aqueous solution studied by pulse radiolysis ${ }^{10}$ and was recently revisited ${ }^{11,12}$.

The product of reaction 1 is also interesting, since it is a neutral atom in a polar solvent, water generally. Such a state, before reacting with other cations, can be stabilized by an atomic induced dipole moment due to the hybridization of atomic orbitals, achieving a large dipole moment. This large dipole moment can then polarize its neighbors. The resulting reaction field enhances the polarizability of the atom. If there is a sufficient number of neighbors, the reaction field can overcome the energy cost of hybridizing the atom entirely and an effective dipolar atom would result. This stabilized state is called excitonic state. The transition to an excitonic phase consisting of dipolar atoms was invoked to understand the metal-nonmetal transition in expanded liquid mercury ${ }^{13}$. Fluid lithium can also undergo a similar transition ${ }^{14}$. Another possibility of having a transition to an excitonic state can occur in a variety of impurity-doped matrix systems, like for example low temperature alkali-doped rare gas solids at low impurity concentration. Such a possibility was described by Logan ${ }^{15}$, considering an alkali atom that, when isolated, contains a single $n s$ electron outside a closed shell. He assumed a sufficiently low impurity concentration, so that the impurity-impurity interactions may be neglected. Such conditions are experimentally possible for these systems ${ }^{16}$. Moreover alkali impurity atoms can be stabilized at low temperature in solid ammonia or in glasses ${ }^{17}$. Liquid host matrices are also possible: Li atom in liquid ammonia presenting the characteristics of an excitonic state has been observed from path integral quantum Monte Carlo calculations of Sprik et al. ${ }^{18,19}$. A similar situation was found by quantum/classical and Car-Parrinello molecular dynamics in the case of the neutral silver atom generated in aqueous solution as in reaction $1^{20}$.

In this review we show our recent progresses addressed to study reaction 1 by means of atomistic simulations and mean-field excitonic state theory, examining in particular the case of the silver atom in water. The case of reaction 2 does not lead to the formation of an excitonic state. It can be computationally studied with 
the same quantum/classical approach, as was done in the case of sodium ${ }^{21}$, but it cannot be interpreted by means of the same mean field theory. Hence, since we are limiting this brief review to the formation of an excitonic state, here we will show only the case of silver, reaction 1 . At this end we employ a mixed quantum/classical molecular dynamics approach based on an adiabatic simulation technique ${ }^{9}$ where the solvated electron is treated quantum-mechanically and the cation and bulk water classically, as described in the next section. Within this approach one can obtain the absorption spectrum of the system, that can be directly compared with experiments and excitonic state theory. Finally, some insights into the formation process are shown. They can be obtained putting together information coming from quantum umbrella sampling of the electron-cation association with free simulation aimed to see the spontaneous reactive process ${ }^{21,22}$.

This brief review is organized as follows. In section 2 we give an overview of the computational techniques adopted to have microscopical information about the processes. Then in section 3 we give a short description of the mean-field formulation of the excitonic state transition and we apply it to the case of silver atom in water comparing theoretical results with information taken from the atomistic simulation and experiments. Finally, in section 4 , the reactive process is investigated. The paper ends with general conclusions of our studies at the present stage and perspectives for future investigations.

\section{Computational approaches}

The systems under investigation are composed by a monovalent cation, an excess electron and solvent water molecules. When the cation is reduced by the electron a neutral atom is formed, otherwise a contact pair or, in a limit case, two noninteracting particles are obtained. The formation and the stability of the neutral atom in water can be studied by MD simulations. Different techniques can be employed, generally following the Born-Oppenheimer approximation. A well established way is to use Car-Parrinello molecular dynamics (CPMD) ${ }^{23}$, where all the interactions are obtained from first principles. Within this approach, one has the advantage that the interactions between particles are not dependent on the system, since no ad hoc parametrization of the potential is needed. Of course, there will be limitations on the dimension of both the system and the time length of the simulations. Another possibility is to divide the system in two parts, one described by classical interactions and the other following the Schrödinger equation. Using this quantum/classical molecular dynamics (QCMD) it is possible to avoid some CPMD limitations, although a parametrization of the potential describing the interactions is needed. In this section we describe the basics of QCMD that can be used to study the reduction of a cation by an electron in solution, while CPMD is a well known method largely presented in literature ${ }^{24}$ and hence we refer the reader to that. At this end we first describe the interactions between the particles of the system - electron, cation and solvent - and then we show the basics of the 
dynamics and finally the quantum umbrella sampling that can be used to study the reaction leading to the cation reduction by the solvated electron.

\subsection{Definition of the interactions}

Before directly addressing the dynamics, a reasonable description of the interactions between the particles is needed. At this end we adopt an additive perspective for the interactions, where the total potential of the system is given by:

$$
V=V_{\mathrm{el}, \mathrm{cat}}+V_{\mathrm{el}, \mathrm{solv}}+V_{\text {cat,solv }}+V_{\text {solv,solv }}
$$

where $V_{\mathrm{el}, \mathrm{cat}}$ and $V_{\mathrm{el}, \text { solv }}$ represent the electron/cation and electron/solvent interactions respectively, $V_{\text {cat,solv }}$ is the cation/solvent interaction potential and $V_{\text {solv,solv }}$ is the solvent/solvent interaction potential. The two former terms are considered as pure classical two-body interactions, composed, as in usual classical force fields, ${ }^{25}$ by a Coulombic and a Lennard-Jones (LJ) term

$$
V_{i j}=\frac{1}{4 \pi \epsilon_{0}} \frac{q_{i} q_{j}}{r_{i j}}+4 \epsilon_{i j}\left[\left(\frac{\sigma_{i j}}{r_{i j}}\right)^{12}-\left(\frac{\sigma_{i j}}{r_{i j}}\right)^{6}\right]
$$

where $i$ and $j$ are two interacting atoms holding to different molecules. For water, the solvent, we adopt the SPC model ${ }^{26}$, while for $\mathrm{Ag}^{+}$/water interactions there were no LJ parameters in the literature, and hence we obtained them ${ }^{21,20}$ in order to better reproduce structural and energetic properties of silver cation in water ${ }^{27}$.

The electron/solvent and electron/cation interaction energies are evaluated adding in the time-independent Schrödinger equation of the electron two pseudopotential terms, leading to

$$
\left(\hat{T}_{e}+\hat{V}_{\mathrm{int}}(\boldsymbol{r}, \boldsymbol{S})\right) \psi_{n}(\boldsymbol{r}, \boldsymbol{S})=E_{n}(\boldsymbol{S}) \psi_{n}(\boldsymbol{r}, \boldsymbol{S})
$$

where $\hat{T}_{e}$ denotes the electronic kinetic energy operator, $\boldsymbol{r}$ the electronic coordinates, $\boldsymbol{S}$ the solvent/cation configurations, $\psi_{n}$ the electronic wavefunction and $\hat{V}_{\text {int }}$ the effective electron-solvent/cation interaction potential. The latter is composed by two contributions, the electron/water, $V_{\mathrm{el} / \text { wat }}$, and the electron/cation, $\hat{V}_{\mathrm{el} / \mathrm{cat}}$, pseudopotentials. For $V_{\mathrm{el} / \text { wat }}$ we use the pseudopotential developed by Turi and Borgis ${ }^{28}$, that is based on a quantum ab initio calculation for one water molecule plus an additional electron confined in a box in the static exchange theory limit. It has the following form

$$
V_{\mathrm{el} / \mathrm{wat}}=V_{\mathrm{SE}}\left(\boldsymbol{r}, \boldsymbol{r}_{\mathrm{O}}, \boldsymbol{r}_{\mathrm{H} 1}, \boldsymbol{r}_{\mathrm{H} 2}\right)+V_{\mathrm{pol}}\left(\boldsymbol{r}, \boldsymbol{r}_{\mathrm{O}}\right)
$$

where $\boldsymbol{r}_{\mathrm{O}}, \boldsymbol{r}_{\mathrm{H} 1}, \boldsymbol{r}_{\mathrm{H} 2}$ are the coordinates of oxygen and hydrogen atoms of water molecules. The first term, $V_{\mathrm{SE}}$, takes into account the interactions of the excess electron with the frozen molecular orbitals of a given water, having the form:

$$
\begin{aligned}
V_{\mathrm{SE}} & =V_{\mathrm{O}}\left(\boldsymbol{r}, \boldsymbol{r}_{\mathrm{O}}\right)+V_{\mathrm{H} 1}\left(\boldsymbol{r}, \boldsymbol{r}_{\mathrm{H} 1}\right)+V_{\mathrm{H} 2}\left(\boldsymbol{r}, \boldsymbol{r}_{\mathrm{H} 2}\right) \\
V_{\mathrm{X}} & =Q_{\mathrm{X}} \frac{\operatorname{erf}\left(A_{\mathrm{X}}\left|\boldsymbol{r}-\boldsymbol{r}_{\mathrm{X}}\right|\right)}{\left|\boldsymbol{r}-\boldsymbol{r}_{\mathrm{X}}\right|}+B_{\mathrm{X}} \frac{\operatorname{erf}\left(C_{\mathrm{X}}\left|\boldsymbol{r}-\boldsymbol{r}_{\mathrm{X}}\right|\right)-\operatorname{erf}\left(D_{\mathrm{X}}\left|\boldsymbol{r}-\boldsymbol{r}_{\mathrm{X}}\right|\right)}{\left|\boldsymbol{r}-\boldsymbol{r}_{\mathrm{X}}\right|}
\end{aligned}
$$


where the index $\mathrm{X}$ represents either $\mathrm{O}$ or $\mathrm{H}, Q_{\mathrm{X}}$ is the partial charge of the molecular sites and $A_{\mathrm{X}}, B_{\mathrm{X}}, C_{\mathrm{X}}$ and $D_{\mathrm{X}}$ are adjustable parameters. The second term, $V_{\mathrm{pol}}$, is a polarization contribution, accounting for electronic correlation effects, for which we use the form proposed by Barnett et al. ${ }^{29}$

$$
V_{\mathrm{pol}}=\frac{\alpha_{\mathrm{W}}}{2\left(\left|\boldsymbol{r}-\boldsymbol{r}_{\mathrm{O}}\right|^{2}+r_{\mathrm{p}}^{2}\right)^{2}}
$$

where $\alpha_{\mathrm{W}}$ is the water molecular polarizability and $r_{\mathrm{p}}$ a typical atomic lenght. For the electron/cation pseudopotential we employed the form proposed by Durand and Barthelat ${ }^{30}$ with a set of refined parameters for silver ${ }^{21}$. It has the form:

$$
\hat{V}_{\mathrm{el}, \mathrm{cat}}=-\frac{1}{r}+\hat{W}^{\mathrm{ps}}
$$

where $-(1 / r)$ is the Coulombic interaction term and $W^{\mathrm{ps}}$ is the nonlocal term of the pseudopotential, given by

$$
\begin{aligned}
\hat{W}^{\mathrm{ps}} & =\sum_{l} W_{l}(r) \hat{P}_{l} \\
W_{l} & =e^{-\alpha_{l} r^{2}} \sum_{i=1}^{n_{l}} c_{i, l} r^{n_{i, l}}
\end{aligned}
$$

where $\hat{P}_{l}$ is the projector on the spherical harmonics $Y_{l m}$. The single electron wavefunctions $\psi_{n}(\boldsymbol{r}, \boldsymbol{S})$ are expanded into a basis of $7 \times 7 \times 7$ spherical Gaussian functions centered on the nodes of a cubic boxes. The form of integrals and the parameters used to represent the electron wavefunctions within the above pseudopotentials were presented previously in detail ${ }^{9,21}$.

\subsection{Quantum/classical molecular dynamics}

Having in hand the interaction potentials described in the previous subsection, it is possible to study the electron/cation/water systems using mixed quantum-classical molecular dynamics (QCMD). We consider each system as composed by two subsystems, a classical subsystem, the cation and the solvent (water), following classical Newton's equation of motion, and a quantum subsystem, the excess electron, following the Schrödinger equation. Note that the electron is not, strictly speaking, treated quantum-dynamically, since we obtain the electron wavefunction solving the time-independent Schrödinger equation 6; anyway for each classical configuration of the solvent/cation subsystem, we have a new electron wavefunction, that contributes to the forces acting on the classical subsystem via the Hellman-Feynman theorem ${ }^{31}$, such that:

$$
\boldsymbol{F}_{S}^{\mathrm{q}}=-\nabla_{\boldsymbol{S}} E_{0}(\boldsymbol{S})=-\int_{-\infty}^{+\infty} \mathrm{d} \boldsymbol{r} \psi_{0}(\boldsymbol{r}, \boldsymbol{S})^{2} \nabla_{\boldsymbol{S}} \hat{V}_{\mathrm{int}}(\boldsymbol{r}, \boldsymbol{S})
$$




\begin{tabular}{|c|c|c|c|}
\hline $\begin{array}{l}\text { Neutral } \\
\text { atom }\end{array}$ & $\begin{array}{c}\text { Excitonic } \\
\text { state }\end{array}$ & $\begin{array}{c}\text { Contact } \\
\text { pair }\end{array}$ & $\begin{array}{c}\text { Separated } \\
\text { species }\end{array}$ \\
\hline 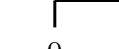 & 1 & T & $T$ \\
\hline
\end{tabular}

Fig. 1. Schematic representation of different states as a function of the electron/cation distance.

In the studies we are reviewing here, we always computed these forces for the ground state of the excess electron $(n=0)$, although an extended usage to excited states is also possible.

For the classical subsystem we immersed the cation in 300 or $800 \mathrm{SPC}$ water molecules, with the excess electron treated with the Born-Oppenheimer dynamics described above. Standard periodic boundary conditions are employed to simulated bulk behaviour with the Ewald summation technique to calculate electrostatic interactions ${ }^{32}$. The simulations are performed in the NVT ensemble using the NoséHoover thermostat to ensure isothermal conditions ${ }^{33}$. The integration of translation and rotation equation of motion is performed using the Gear predictor-corrector algorithm ${ }^{34}$, fixing the time-step at $0.5 \mathrm{fs}$, and the simulations runs have a typical time length of a few tens of picoseconds after a stabilization time.

\subsection{Reaction free energy profile}

The cation reduction reaction path can be studied obtaining, from QCMD simulations, free energy curves as a function of the electron/cation distance, $r_{n}(\boldsymbol{S})$, defined as

$$
r_{n}(\boldsymbol{S})=\left|\left\langle\Psi_{n}|\boldsymbol{r}| \Psi_{n}\right\rangle-\boldsymbol{R}_{c a t}\right|=\xi
$$

The distance $\xi$ is the easiest microscopic property given by simulations we can relate to the formation of an excitonic state, coupled with a wavefunction analysis. The limiting situation $\xi=0$ corresponds to the electron located on the atom. Increasing $\xi$, we have first a distortion of the atom leading to a dipolar atom (the excitonic state, largely described in the next section), then an electron/cation contact pair is formed, while for larger values of $\xi$ we have two distinct solvated species, the hydrated electron and the cation (see fig 1).

Along this coordinate, the free energy function will be given by

$$
F_{n}(\xi)=-k_{\mathrm{B}} T \ln P_{n}(\xi)
$$

where $P_{n}(\xi)$ is the probability to find the electron in its electronic state $n$ for the electron/cation distance $\xi$,

$$
P_{n}(\xi)=\left\langle\delta\left(r_{n}(\boldsymbol{S})-\xi\right)\right\rangle_{n}
$$


The solvent average $\langle\cdots\rangle_{n}$ and the data points $r_{n}(\boldsymbol{S})$ for the reaction coordinate are calculated by QCMD with the total Hamiltonian

$$
H_{n}(\boldsymbol{S})=E_{n}(\boldsymbol{S})+\mathcal{H}(\boldsymbol{S})
$$

where $\mathcal{H}(\boldsymbol{S})$ is the classical kinetic and potential energy due to the solvent and the cation. To sample the needed electron/cation distances we use the Umbrella Sampling (US) approach, adding the following quadratic potential to the Hamiltonian of equation 18:

$$
U_{\xi_{0}}=\frac{1}{2} k\left(r_{n}(\boldsymbol{S})-\xi_{0}\right)^{2}
$$

The US technique was adapted for the case of a system including a quantum particle by Borgis and Staib ${ }^{35}$ and this methodology was succesfully used in the case of cations reduction process ${ }^{21,22}$.

\section{Dipolar excitonic state}

In this section we briefly review the basic concepts of the mean field dipolar excitonic state theory based on works of Logan and coworkers ${ }^{15,36}$ with a particular focus on the application to a neutral atom in a liquid host matrix. Notably the case of silver in water is analyzed looking for its properties. In particular from excitonic state theory it is possible to obtain the hybridized atomic orbitals and hence the atomic wavefunctions of the states considered. In this way the induced silver dipole and electronic transitions between $s$ and $p$ states can be evaluated and directly compared with QCMD results and experimental data.

\subsection{General theory}

Considering an atom containing a single $n s$ electron outside a closed shell, like elements of groups 1 (alkali atoms) and 11 (for example silver), it is possible to write the isolated atom Hamiltonian considering simply a single $n s$ orbital with energy $\epsilon_{0}=-\hbar \omega_{0}$ and three $n p$ orbitals, with energy $\epsilon_{1}=\hbar \omega_{0}$. Neglecting higher excited states, the atomic Hamiltonian is therefore reduced to a simple four level system (FLS) that can be represented by

$$
\hat{H}_{0}=\hbar \omega_{0}\left(-|s\rangle\left\langle s\left|+\sum_{\alpha}\right| p_{\alpha}\right\rangle\left\langle p_{\alpha}\right|\right)
$$

where $\alpha=x, y$ or $z$. An external electric field will introduce the perturbation $\hat{H}_{E}=-\hat{\boldsymbol{\mu}} \cdot \mathbf{E}^{\mathbf{0}}$, where $\hat{\boldsymbol{\mu}}$ is the dipole moment operator. In the above basis the perturbation Hamiltonian is given by

$$
\hat{H}_{E}=\hbar \sum_{\alpha}\left(|s\rangle\left\langle p_{\alpha}|+| p_{\alpha}\right\rangle\langle s|\right) \xi_{\alpha}
$$

where $\hbar \xi_{\alpha}=M E_{\alpha}^{0}$ represents the energy contribution due to the external field and $M=\left\langle s\left|\hat{\mu}_{\alpha}\right| p_{\alpha}\right\rangle$ is the transition dipole moment. The new Hamiltonian, $\hat{H}_{0}+\hat{H}_{E}$, 
can be diagonalised leading to four eigenvalues, $\hbar \omega_{-}^{0}, \hbar \omega_{0}$ (doubly degenerate) and $\hbar \omega_{+}^{0}$, where

$$
\omega_{ \pm}^{0}= \pm \sqrt{\omega_{0}^{2}+\xi^{2}}
$$

and the corresponding normalized, orthogonal eigenfunctions are given by

$$
\begin{aligned}
\left|\Psi_{-}^{0}\right\rangle & =\left|s+\lambda p_{0}\right\rangle\left[1+\lambda^{2}\right]^{-1 / 2} \\
\left|\psi_{0}^{0}\right\rangle & =\left|p_{1}\right\rangle \text { and }\left|p_{-1}\right\rangle \\
\left|\psi_{+}^{0}\right\rangle & =\left|p_{0}-\lambda s\right\rangle\left[1+\lambda^{2}\right]^{-1 / 2}
\end{aligned}
$$

where

$$
\lambda=\frac{\left[\left(\omega_{0}^{2}+\xi^{2}\right)^{1 / 2}-\omega_{0}\right]}{|\xi|}
$$

Since the new (perturbed) wavefunctions are obtained, one can express the new (perturbed) transition dipole moments in this basis, giving

$$
\begin{aligned}
\left\langle\Psi_{-}^{0}|\hat{\boldsymbol{\mu}}| \psi_{+}^{0}\right\rangle & =\frac{1-\lambda^{2}}{1+\lambda^{2}} \mathbf{M} \\
\left\langle\Psi_{-}^{0}|\hat{\boldsymbol{\mu}}| \psi_{0}^{0}\right\rangle & =\frac{1}{\left(1+\lambda^{2}\right)^{1 / 2}} \mathbf{M}
\end{aligned}
$$

We can now consider that the atom (the impurity) is immersed in an inert (non reactive) condensed phase matrix, solid or liquid. Assuming a sufficiently low concentration of the impurity, we can neglect impurity-impurity interactions, thus considering a single matrix-bound impurity. The mean field theory we are going to shortly review here is based on the following assumptions: (i) the impurity atom valence electron remains strongly localized; (ii) the $n s \rightarrow n p$ excitations in the matrix-bound impurity are of Frenkel type; (iii) there is no overlap with the host matrix absorption bands.

Following the continuum dielectric theory ${ }^{37}$, we can express the local field acting on the matrix-bound impurity, $\boldsymbol{f}(\boldsymbol{E})$, as given by two contributions:

$$
\boldsymbol{f}(\boldsymbol{E})=\boldsymbol{G}(\boldsymbol{E})+\boldsymbol{R}(\boldsymbol{E} ;\langle\boldsymbol{\mu}\rangle)
$$

where $\boldsymbol{G}(\boldsymbol{E})$ is the electric field inside the cavity due to external sources and $\boldsymbol{R}(\boldsymbol{E} ;\langle\boldsymbol{\mu}\rangle)$ is the reaction field due to the total impurity dipole moment, $\boldsymbol{\mu}$, that polarizes its sourronding. Hence, the mean field interaction Hamiltonian, $\hat{H}_{i n t}$, can be expressed as

$$
\begin{aligned}
\hat{H}_{\mathrm{int}} & =-\hat{\boldsymbol{\mu}} \cdot \boldsymbol{f}(\boldsymbol{E}) \\
& =-\hat{\boldsymbol{\mu}} \cdot\left[\frac{3 \epsilon_{m}}{2 \epsilon_{m}+1} \boldsymbol{E}+g\left(\rho_{m}\right)\langle\boldsymbol{\mu}\rangle\right]
\end{aligned}
$$


where $g\left(\rho_{m}\right)$ is the reaction field factor

$$
g\left(\rho_{m}\right)=\frac{8 \pi \rho_{m}}{3} \frac{\left[\epsilon_{m}-1\right]}{\left[\epsilon_{m}+1\right]}
$$

with $\rho_{m}$ and $\epsilon_{m}$ being the matrix density and dielectric constant respectively. Hence, the effective Hamiltonian, $\hat{H}_{\mathrm{i}}$, will be given by

$$
\hat{H}_{\mathrm{i}}=\hat{H}_{0}+\hat{H}_{\mathrm{int}}
$$

To obtain $\hat{H}_{\text {int }}$ from equation 31 we should know $\langle\boldsymbol{\mu}\rangle$, the impurity dipole moment, that, as the expectation value of any quantum operator, is given by

$$
\langle\boldsymbol{\mu}\rangle=Q_{i}^{-1} \operatorname{Tr}\left\{\hat{\boldsymbol{\mu}} \exp \left[-\beta \hat{H}_{i}(\langle\boldsymbol{\mu}\rangle)\right]\right\}
$$

where $\operatorname{Tr}$ is over the eigenstates of $\hat{H}_{i}=\hat{H}_{0}+\hat{H}_{\text {int }}$.

We have hence a self-consistent equation for the matrix-bound impurity dipole moment. The solution for $\langle\boldsymbol{\mu}\rangle$ will be a function of both the atomic properties of the free impurity and the thermodynamic properties of the host matrix, i.e. the environment. In the absence of an applied external electric field, $(\boldsymbol{E}=0)$, the self-consistent equation for $\langle\boldsymbol{\mu}\rangle$ is

$$
\langle\boldsymbol{\mu}\rangle_{0} y\left(\langle\mu\rangle_{0}\right)=\langle\boldsymbol{\mu}\rangle_{0} \frac{\tanh \left[\beta s\left(\rho_{m}\right) y\left(\langle\mu\rangle_{0}\right)\right]}{1+\exp \left[-\beta \hbar \omega_{0}\right] \operatorname{sech}\left[\beta s\left(\rho_{m}\right) y\left(\langle\mu\rangle_{0}\right)\right]}
$$

with

$$
y\left(\langle\mu\rangle_{0}\right)=\left[\alpha_{0} g\left(\rho_{m}\right)\right]^{-1} \sqrt{1+\frac{\alpha_{0}}{\hbar \omega_{0}}\left[g\left(\rho_{m}\right)\langle\mu\rangle_{0}\right]^{2}}
$$

where $\alpha_{0}$ is the polarizability of the FLS and

$$
s\left(\rho_{m}\right)=\hbar \omega_{0} \alpha_{0} g\left(\rho_{m}\right)>0
$$

Note that to solve equation 35 one has only to know properties that characterize the isolated impurity atom, $\alpha_{0}$ and $\omega_{0}$, and the host matrix, $\rho_{m}$ and $\epsilon_{m}$. It is evident that one possible solution of equation 35 is the trivial one, $\langle\boldsymbol{\mu}\rangle_{0}=0$, corresponding to the absence of an atomic dipole. Moreover, given the atomic properties $\alpha_{0}$ and $\omega_{0}$, at a fixed temperature, exists a critical density, $\rho_{m, c}$ such that for $\rho_{m}<\rho_{m, c}$ only the trivial solution $\langle\boldsymbol{\mu}\rangle_{0}=0$ can fulfil the self-consistent equation 35 . On the other hand, for $\rho_{m}>\rho_{m, c}$ two solutions are possible, $\langle\boldsymbol{\mu}\rangle_{0}=0$ and $\langle\boldsymbol{\mu}\rangle_{0}>0$, where the latter is favoured thermodynamically. Hence, for $\rho_{m}=\rho_{m, c}$ the system has a transition to a dipolar excitonic state, $\langle\boldsymbol{\mu}\rangle_{0}>0$.

A simplified expression of the self-consitent equation 35 can be obtained for the limit situation $y\left(\langle\mu\rangle_{0}\right) \rightarrow 1$, leading to

$$
\frac{\langle\mu\rangle_{0}^{2}}{M^{2}}=\frac{\left[\alpha_{0} g\left(\rho_{m}\right)\right]^{2}-1}{\left[\alpha_{0} g\left(\rho_{m}\right)\right]^{2}}
$$

that preserves all the features of a transition to a dipolar excitonic state, and that was conveniently used to analyze the presence of a dipolar excitonic state in liquid systems 19,20 . 


\subsection{Dipolar atom properties}

Once the excitonic state is obtained, the original atomic properties will of course be changed. This changes can be thought in terms of hybridization of original $s$ and $p$ orbitals, leading to the wave-function

$$
|\Psi(\tilde{\lambda})\rangle=\left|S+\tilde{\lambda} P_{z}\right\rangle\left[1+\tilde{\lambda}^{2}\right]^{-1 / 2}
$$

and the $s p$-hybrid state will have the following dipole moment

$$
\begin{aligned}
\left\langle\mu_{\alpha}\right\rangle_{0} & =\left\langle\Psi(\tilde{\lambda})\left|\hat{\mu}_{\alpha}\right| \Psi(\tilde{\lambda})\right\rangle \\
& =\frac{2 \tilde{\lambda} M}{\left[1+\tilde{\lambda}^{2}\right]} \delta_{\alpha z}
\end{aligned}
$$

where $\tilde{\lambda}$ is a mixing coefficient that here can be regarded as a parameter. It will be useful to express the total free energy change of the system associated to the formation of the dipolar atomic state as a function of $\tilde{\lambda}$, taking as reference the normal state, $\langle\boldsymbol{\mu}\rangle_{0}=0$, where $\tilde{\lambda}=0$. This total free energy change, $\Delta A(\tilde{\lambda})$, is composed by two contributions: (i) the hybridization free energy cost, $A_{h}(\tilde{\lambda})$, and (ii) the free energy arising from the interaction with the environment, $A_{s}(\tilde{\lambda})$. Then the total free energy change, $\Delta A(\tilde{\lambda})=A_{h}(\tilde{\lambda})+A_{s}(\tilde{\lambda})$, will be given by

$$
\Delta A(\tilde{\lambda})=2 \hbar \omega_{0} \frac{\tilde{\lambda}^{2}}{\left[1+\tilde{\lambda}^{2}\right]}\left\{1-\frac{\alpha_{0} g\left(\rho_{m}\right)}{\left[1+\tilde{\lambda}^{2}\right]}\right\}
$$

Note that for $\rho_{m}<\rho_{m, c} \Delta A(\tilde{\lambda})$ has a minimum at $\tilde{\lambda}=0$, i.e. the normal state is the stable state; on the other hand for $\rho_{m} \geqslant \rho_{m, c}, \Delta A(\tilde{\lambda})$ has a minimum for

$$
\lambda^{2}=\frac{\left[\alpha_{0} g\left(\rho_{m}\right)-1\right]}{\left[\alpha_{0} g\left(\rho_{m}\right)+1\right]}
$$

Once the dipolar excitonic state is formed $\left(\rho_{m}>\rho_{m, c}\right)$ the energies of the perturbed state described by $\left|\Psi_{-}\right\rangle$and $\left|\psi_{+}^{0}\right\rangle$ in equations 23 and 25 will be splitted by

$$
\Delta_{E I}=\omega_{0}\left(\left\{1+\frac{\alpha_{0}}{\hbar \omega_{0}}\left[g\left(\rho_{m}\right)\langle\mu\rangle_{0}\right]^{2}\right\}^{1 / 2}-1\right)
$$

such that

$$
\omega_{ \pm}= \pm\left(\omega_{0}+\Delta_{E I}\right)
$$

Atomistic simulations can be seen in the spirit of this theory looking, e.g., for properties related to the impurity atom that can be directly addressed by them. In this way one can interpret microscopic observations taken from simulations in terms of a mean-field theory based on experimental observables (i.e. an essentially macroscopic theory). In particular the average dipole moment of the impurity can be obtained from MD simulations. This study was done originally by Logan 19 
analyzing a simulation study of $\mathrm{Li}$ in ammonia of Sprik et al. ${ }^{18}$, where he pointed out that the simple equation 38 can predict the formation of an atomic dipole of Li obtaining a good quantitative agreement with results found in the atomistic simulation. More recently, the behaviour of neutral silver atom in water was studied by two indipendent and different atomistic simulations, CPMD and QCMD ${ }^{20}$. Also in this case a neutral dipolar atom is formed, i.e. an excitonic state, providing a good agreement between theory and simulations (notably $1.96 \mathrm{D}$ from the theory versus $1.9 \mathrm{D}$ and $2.4 \mathrm{D}$ obtained from CPMD and QCMD respectively). This state is particulary interesting also from the point of view of the solvent structures. In fact it mainly presents the typical features of an hydrophobic species, like similar oxygen and hydrogen peaks in the electron/water radial distribution function, but it is in part organized to stabilize the atomic induced dipole, that adiabatically follows the electric field applied by the solvent ${ }^{20}$. As already pointed the silver cation is reduced by the electron in water solution leading to a dipolar atom, that can be well interpreted as an excitonic state. From the same kind of atomistic simulations we also noticed that replacing silver cation with sodium cation a different situation is obtained ${ }^{21}$. Changing the chemical nature of the cation, sodium now, it is no more reduced, as expected, and only a metastable cation/electron contact pair is formed with an average distance of $\sim 2 \AA$. This metastable contact pair was also found by Laria in micelles ${ }^{38}$ and its presence was suspected by experiments ${ }^{39}$ - of course being a metastable state, it is experimentally difficult to be detected. In this case the basic assumptions of the excitonic theory are no more valid, for example simply because the $s$ electron is no more localized on the atom.

\subsection{Electronic transitions}

Systems where an excess electron is formed in solution in presence of cations are generally investigated experimentally via electronic spectroscopy ${ }^{40,12}$. It is evident, from equations 23-25 and 44- 45, that excitonic theory is in principle able to provide transition energies between $n s$ and $n p$ states of the impurity. To fully investigate the spectroscopic consequences of a transition to an excitonic state, at least two effects must be incorporated in the theory: the spin orbit coupling interaction and the spatially local, primarly repulsive, interactions (corresponding to the usual van der Waals classical repulsive short range interactions). Hence, the Hamiltonian $\hat{H}_{i}$ of equation 33 is replaced by

$$
\hat{H}_{i n t}^{\prime}=\hat{H}_{i}+\hat{H}_{s o}+\hat{V}_{0}
$$

where $\hat{H}_{s o}$ is the spin-orbit coupling Hamiltonian and $\hat{V}_{0}$ is a spherically simmetric interaction term taking into account the local interactions that do not mix the $n s$ and $n p$ orbitals.

Then, it is possible to write simple expressions for the total resulting transition energies 


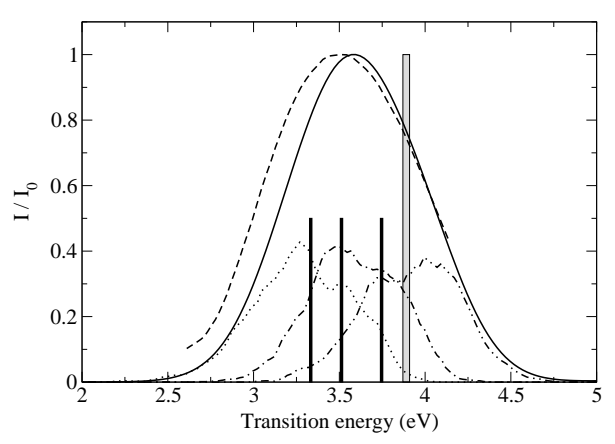

Fig. 2. Absorption spectrum of silver atom in bulk water: experimental (dashed line), calculated from QCMD simulations (solid line) and obtained from excitonic state theory (vertical black lines). The three components obtained from QCMD simulations are separately shown (dotted, dasheddotted and dashed-dotted-dotted) corresponding to $s \rightarrow p$ transitions. The in vacuum transition is also shown (vertical open line).

$$
\begin{aligned}
& \Delta E_{1}=E_{0}+\delta+\frac{3}{2} \Delta_{E I}-\frac{1}{4} \zeta-\frac{1}{2}\left[\Delta_{E I}^{2}+\zeta \Delta_{E I}+\frac{9}{4} \zeta^{2}\right]^{1 / 2} \\
& \Delta E_{2}=E_{0}+\delta+\Delta_{E I}+\frac{1}{2} \zeta \\
& \Delta E_{3}=E_{0}+\delta+\frac{3}{2} \Delta_{E I}-\frac{1}{4} \zeta+\frac{1}{2}\left[\Delta_{E I}^{2}+\zeta \Delta_{E I}+\frac{9}{4} \zeta^{2}\right]^{1 / 2}
\end{aligned}
$$

where $E_{0}=2 \hbar \omega_{0}, \zeta$ is an effective $n p$ spin-orbit coupling constant and $\delta$ is the energy shift between $n s$ and $n p$ orbitals due to $\hat{V}_{0}$ (i.e. $\delta=\left\langle p\left|\hat{V}_{0}\right| p\right\rangle-\left\langle s\left|\hat{V}_{0}\right| s\right\rangle$ ). Note that equations 47-49 contain the normal state $\left(\Delta_{E I}=0, \Delta E_{2}=\Delta E_{3}\right)$ and the excitonic state $\left(\Delta_{E I} \neq 0\right)$.

To directly address the transition energies from equations $47-49, \delta$ is probably the most problematic term to estimate. It is also a crucial value, since, disregarding it, the theory can provide only blue-shifted spectra. If a red-shifted spectrum is observed, it can be justified by a negative $\delta$ that bilances a positive $\Delta_{E I}$. Unfortunately, $\delta$ cannot be obtained analitically but only via semiempirical considerations. In the case of silver, the explicit case under our interest, we can make a first drastic, but reasonable, assumption considering $\delta_{p}<<\delta_{s}$ and then $\delta=-\delta_{s}$. This term, $\delta_{s}$, can be further estimated from the energy of $|S\rangle$ state in vacuo, $E_{v a c}^{|S\rangle}$, and in solution, $E_{\text {sol }}^{|S\rangle}$, as obtained from simulations reported in ref. ${ }^{21}$, and using $\Delta_{E I}$ via equation 44 as the electrostatic contribution of the solvent. Thus,

$$
\delta_{s}=E_{s o l}^{|S\rangle}-E_{v a c}^{|S\rangle}+\hbar \Delta_{E I}
$$

The spin orbit contribution can be taken from recent experimental data ${ }^{41}$. Using the values listed in $\operatorname{tab} 1$ for $\mathrm{Ag}^{0}$ in water, we can obtain the three transition energies: $\Delta E_{1}=3.33 \mathrm{eV}(\equiv 372 \mathrm{~nm}), \Delta E_{2}=3.51 \mathrm{eV}(\equiv 353 \mathrm{~nm})$ and $\Delta E_{3}=$ 
Table 1. Parameters adopted and values obtained to study the excitonic state of silver atom in water at ambient conditions. ${ }^{(a)}$ experimental value from ref. ${ }^{41} ;{ }^{(b)}$ calculated from equation 44 ; (c) calculated from equation 50 .

\begin{tabular}{lr}
\hline Property & Value \\
\hline$\alpha_{A g}$ & $7.9 \AA^{3}$ \\
$\mathrm{M}_{A g}$ & $4.8 \mathrm{D}$ \\
$\omega_{0}$ & $15082.9 \mathrm{~cm}^{-1(a)}$ \\
$\zeta$ & $613.8 \mathrm{~cm}^{-1(a)}$ \\
$\epsilon_{m}$ & 78.3 \\
$\rho_{m}$ & $1.000 \mathrm{~g} / \mathrm{cm}^{3}$ \\
$\Delta_{E I}$ & $1380.3 \mathrm{~cm}^{-1(b)}$ \\
$\delta_{s}$ & $3548.9 \mathrm{~cm}^{-1(c)}$ \\
\hline
\end{tabular}

$3.75 \mathrm{eV}(\equiv 331 \mathrm{~nm})$. All these values are in good agreement with experimental observations ${ }^{12}$. Moreover from QCMD simulations we can obtain as well the overall spectrum of $\mathrm{Ag}^{0}$ in water that is in good agreement with experiments and also with the previous theoretical considerations (see figure 2).

\section{Formation reactions}

In this section we show how it is possible to obtain via atomistic simulations some information on the reactive process between the solvated electron and a cation in water solution. Generally speaking, one can be interested in equilibrium thermodynamics or kinetics properties. In the former case the quantum Umbrella Sampling described in section 2.3 can be used in order to have the free energy profile of the reaction. This is useful mainly to locate the stable state along an electron/cation distance coordinate, as defined in equation 15. As expected, this free energy curve presents a clear minimum for electron/silver distance $\sim 0.5 \AA$, as shown in figure 3 (for comparison, the free energy curve for a sodium cation is also plotted). This corresponds to the average distance obtained from unconstrained simulations of the excitonic state described previously. It is also possible to show, from a simple thermodynamic cycle, that the free enthalpy of reduction, $\Delta \mathrm{G}^{*}$, is $+0.96 \mathrm{eV}$ and $-0.74 \mathrm{eV}$ for sodium and silver respectively ${ }^{22}$. In addition, another important thermodynamic information comes out from umbrella sampling calculations, namely the free energy barrier to escape from the stable state. In the case of silver it was interesting to note that the atomic excitonic state presents a high barrier (over $40 k_{B} T$ ), confirming that this state is both thermodynamically and kinetically stable.

Unfortunately, a full quantitative kinetic information cannot be directly addressed from these curves, since they correspond to an equilibrium solvation regime ${ }^{42}$ that assumes the solvent instantaneously relaxing into an equilibrium state. As a consequence, they lack a solvent coordinate that, consistently with Mar- 


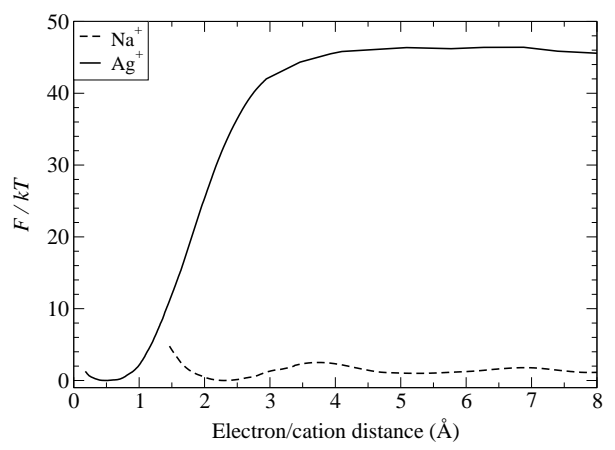

Fig. 3. Free energy profiles as a function of electron-cation distance obtained for silver (solid) and sodium (dashed) cations.

cus theory for electron transfer ${ }^{1}$, should be employed to fully describe the reaction process. Moreover, as we noted recently, the barrier high changes as a function of the number of water molecules considered ${ }^{22}$, preventing us from extracting quantitative kinetic information.

Despite of the previously mentioned limitations, the choise of the electron/cation distance to describe the free energy profile of the reactive process has some advantages. As already noticed, we can determine which is the thermodynamically stable state from the same intuitive definition done in terms of electron/cation distance used in the previous sections. It is also useful since an excitonic stable state can come out directly and it can be related to equilibrium properties of other intermediate states. Moreover, it can be related to experiments done with different cation concentrations leading to different stationary absorption spectra. ${ }^{39,43,12}$

As already remarked, from these curves a full kinetic information cannot be directly addressed. Whereas a large barrier can tell us, qualitatively, that the thermal reaction leading to a separated cation/electron state is kinetically unfavoured, a quantitative calculation of kinetic constants needs more effort. Anyway, in the present case we can be sufficiently convinced that this state is kinetically stable other than thermodynamically. Doing independent free QCMD simulations at different starting distances $d_{0} \geqslant 4 \AA$, we can obtain some insights into the reduction process (see figure 4). In fact, it is possible to decompose the silver reduction process in the following steps: (i) a diffusive (brownian) step where the electron/cation distance reaches a value of $\sim 4 \AA$, (ii) a solvent/solute organization step that allows the decrease of distance down to $\sim 1 \AA$, occurring in $\sim 0.15$ ps and (iii) a relatively slow decrease of this distance until the formation of the excitonic state, occurring in $\sim 0.8$ ps. Note that the time scale of the non diffusive processes we identified above (i.e. the last two steps) are in agreement with the experimental hypothesis that the reaction should occurs in less than 1 ps ${ }^{12}$.

To identify the nature of the system between steps (ii) and (iii), that we will 

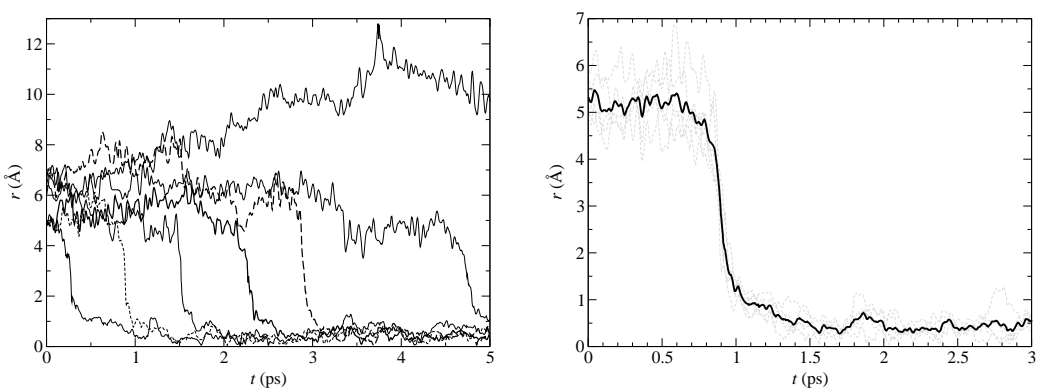

Fig. 4. Electron/cation distance as a function of time during unconstrained simulations of the reduction process. On the right panel, trajectories have been shifted in time; the bold curve is the mean of all trajectories.

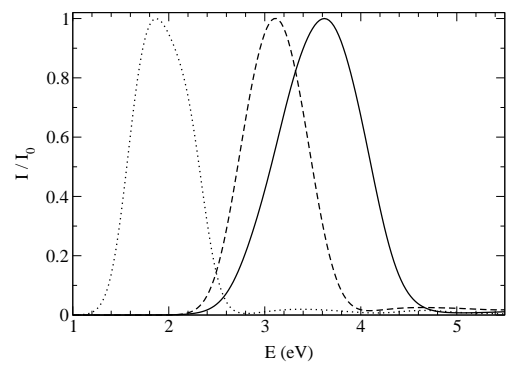

Fig. 5. Absorption spectrum of the excess electron in the reduction intermediate state (dashed line), compared to the Ag excitonic state (solid line) and the free hydrated electron (dotted line).

call the reduction intermediate state, we obtained its absorption spectrum (figure 5) and the radial density distributions around silver (figure 6) by an average over the first 200 fs of step (iii). Comparing the spectrum with those of a free hydrated electron and of the silver excitonic state, we see that its maximum is close to the silver excitonic state, while its width is closer to the free hydrated electron. Moreover, the study of radial density distributions reveals that this intermediate state has a weak solvation structure, with oxygen atoms still ordered around the silver but hydrogen atoms with less defined position. We interpret these results as a rotational reorganization of the solvation structure, with water molecules still presenting a configuration reminiscent of the cation solvation structure. Thus, we can think that the electron rapid jump on the silver cation (step (ii)) is initiated by a favorable configuration of the fluctuating orientation of water molecules around the cation. 


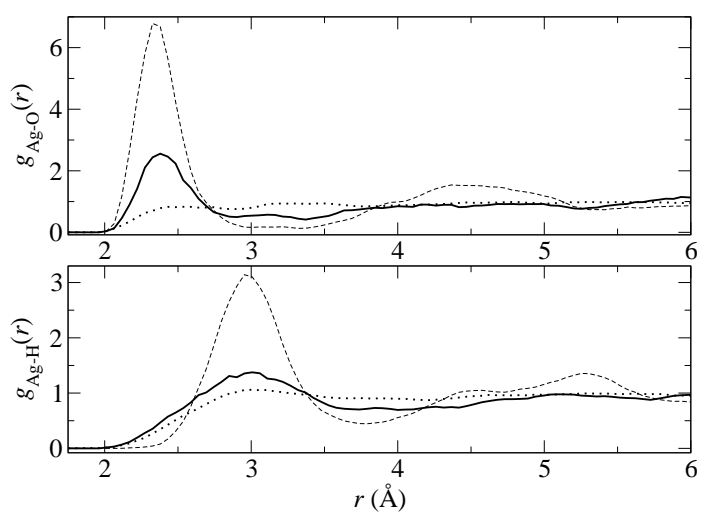

Fig. 6. Radial density functions for oxygen (upper panel) and hydrogen (lower panel) around the silver atom in the reduction intermediate state (thick line), compared to the silver excitonic state (thin line) and the $\mathrm{Ag}^{+}$cation (dashed line).

\section{Conclusions and perspectives}

Here, we have reviewed our recent progresses in understanding the formation and stability of neutral atoms in water. In particular, we have shown as from atomistic simulations and theoretical analysis it is possible to better understand the physico-chemical basis of such phenomena. At this end we have studied the simplest reduction process, i.e. the direct interaction between a cation, the reducible species, and the electron, the reducent, in solution. We have found, in the case of silver, that the stationary state we obtained from simulations, the excitonic state, was in good agreement with experimental studies, done in the same thermodynamic conditions, and theoretical analysis. In particular, atomistic simulations are able to well reproduce experimental UV-Vis spectra of such systems. This is particulary encouraging in order to extend the research to other similar systems, like other cations in solution and in confined systems. At this end, the study of cations reduction by pulse radiolysis in zeolites is an intriguing perspective, that could help to better understand recent advanced experiments (REF). Moreover, the identification of this excitonic state is particularly interesting since it allows us to analyze it also via theoretical formulation. This analysis is particulary important because we can have another confirm of the good quality of the data obtained from simulations and we can be able to rationalize them in terms of physical macroscopic conditions.

Furthermore, we have shown that studying this kind of systems by means of mean field excitonic theory it is also useful to understand some spectroscopic data, obtaining a nice agreement between experiments, theory and simulations. This result can give us the basis to investigate with a sufficient degree of confidence the formation of such neutral atoms in different thermodynamic states (of particular interest can be the supercritical conditions) and in perspective it can be also used to predict the spectroscopic behaviour of other atoms. 
Finally, we have shown as we can have a general picture of the overall reactive processes, from both constrained - the umbrella sampling studies - and unconstrained simulations. This gives us a first picture of the elementary reduction reaction. To better understand this point, we are going to complete the studies we have done with other techniques aimed to simulate complex reactions (REF) and also, from the theoretical point of view, with a more detailed study of non-equilibrium solvation effects.

\section{Acknowledgements}

We wish to acknowledge C. Nicolas, R. Vuilleumier and P. Archirel for fruitful collaborations in this project and D. Borgis, M. Mostafavi, B. Lévy and A. H. Fuchs for several interesting discussions. We thank the Institut du Développement et des Ressources en Informatique Scientifique (IDRIS) of the CNRS for generous allocations of computer time and the CNRS/DFG and European Union through Marie Curie project for fundings.

\section{References}

1. R. A. Marcus, J. Chem. Phys. 24, 966 (1956); R. A. Marcus and N. Sutin, Biochim. Biophys. Acta 811, 265 (1985); M. D. Newton and N. Sutin Ann. Rev. Phys. Chem. 35, 437 (1984).

2. J. H. Baxendale and F. Busi, The Study of fast Processes and transient Species by Electron Pulse Radiolysis, NATO ASI Series 86 (D. Reidel Publishing Co., Dordrecht 1982).

3. E. J. Kirschke and W. L. Jolly, Inorg. Chem. 6, 866 (1967); O. H. Leblanc, J. Chem. Phys. 30, 1443 (1959); J. Belloni, G. van Amerongen, M. Herlem, J. L. Sculfort and R. Heindl, J. Phys. Chem. 84, 1269 (1980); R. Laenen and T. Roth, J. Mol. Struct. 598, 37 (2001).

4. E. J. Hart and J. W. Boag, J. Am. Chem. Soc. 84, 4090 (1962).

5. J. Jortner, J. Chem. Phys. 27, 823 (1957); J. Jortner, J. Chem. Phys. 30, 839 (1959).

6. E. J. Hart, B. D. Michael and K. H. Schmidt, J. Phys. Chem. 75, 2798 (1971); H. Christensen and K. Sehested, J. Phys. Chem. 90, 186 (1986); G. Wu, Y. Katsumura, Y. Muroya, X. Li and Y. Terada, Chem. Phys. Lett. 325, 531 (2000).

7. A. Migus, Y. Gaudel, J. L. Martin and A. Antonetti, Phys. Rev. Lett. 58, 1559 (1987); F. H. Long, H. Lu and K. B. Eisenthal, Phys. Rev. Lett. 64, 1469 (1990); C. Silva, P. K. Walhout, K. Yokoyama and P. F. Barbara, Phys. Rev. Lett. 80, 1086 (1998); M. Assel, R. Laenen and A. Laubereau, J. Chem. Phys. 111, 6869 (1999); M. Assel, R. Laenen and A. Laubereau, J. Phys. Chem. A 102, 2256 (1998); M. Assel, R. Laenen and A. Laubereau, Chem. Phys. Lett. 317, 13 (2000).

8. B. J. Schwartz and P. J. Rossky, J. Chem. Phys. 101, 6902 (1994); B. J. Schwartz and P. J. Rossky, J. Chem. Phys. 101, 6917 (1994); B. J. Schwartz and P. J. Rossky, Phys. Rev. Lett. 72, 3282 (1994); D. Borgis and S. Bratos, J. Mol. Struct. 436-7, 537 (1997); M. Boero, M. Parrinello, K. Terakura, T. Ikeshoji and C. C. Liew, Phys. Rev. Lett. 90, 226403 (2003).

9. C. Nicolas, A. Boutin, B. Lévy and D. Borgis, J. Chem. Phys. 118, 9689 (2003).

10. J. H. Baxendale, E. M. Fielden, J. P. Keene and M. Ebert, in Pulse Radiolysis, J. P. Keene, A. Swallow, J. H. Baxendale Eds., 207-220 (Academic Press, London 1965); 
R. Tausch-Treml, A. Henglein and J. Lilie, Ber. Bunsenges. Phys. Chem. 82, 1343 (1978); J. Von Pukies, W. Roebke and A. Henglein, Ber. Bunsenges. Phys. Chem. 72, 842 (1968); M. Mostafavi, J. L. Marignier, J. Amblard and J. Belloni, Radiat. Phys. Chem. 34, 605 (1989).

11. P. Mulvaney and A. Henglein, Chem. Phys. Lett. 168, 391 (1990); E. Janata, A. Henglein and B. G. Ershov, J. Phys. Chem. 98, 10888 (1994); E. Janata, J. Lilie and M. Martin, Rad. Phys. Chem. 43, 353 (1994).

12. M. Mostafavi, M. Lin, G. Wu, Y. Katsumura and Y. Muroya, J. Phys. Chem. A 106, 3123 (2002).

13. W. Hafner and F. Hensel, Phys. Rev. Lett. 48, 1026 (1982); L. A. Turkevich and M. H. Cohen, Phys. Rev. Lett. 53, 2323 (1984); L. A. Turkevich and M. H. Cohen, J. Phys. Chem. 88, 3751 (1984).

14. D. E. Logan and P. P. Edwards, Philos. Mag. B 53, L23 (1986); D. E. Logan and P. P. Edwards, Ber. Bunsenges. Phys. Chem. 90, 575 (1986).

15. D. E. Logan, J. Chem. Phys. 86, 234 (1987).

16. N. Schwentner, E.-E. Koch and J. Jortner, Electronic Excitations in Condensed Rare Gases (Springer, Heidelberg, 1985).

17. P. F. Meier, R. H. Hauge and J. L. Margrave, J. Am. Chem. Soc. 100, 2108 (1978); R. Catterali and P. P. Edward, Chem. Phys. Lett. 42, 540 (1976).

18. M. Sprik, R. W. Impey and M. L. Klein, Phys. Rev. Lett. 56, 2326 (1986).

19. D. R. Logan, Phys. Rev. Lett. 57, 782 (1986).

20. R. Spezia, C. Nicolas, A. Boutin and R. Vuilleumier, Phys. Rev. Lett. 91, 208304 (2003).

21. R. Spezia, C. Nicolas, P. Archirel and A. Boutin, J. Chem. Phys. 120, 5261 (2004).

22. R. Spezia, C. Nicolas, F.-X. Coudert, P. Archirel, R. Vuilleumier and A. Boutin, Mol. Simulat., in press.

23. R. Car and M. Parrinello, Phys. Rev. Lett. 55, 2471 (1985); J. Hutter, A. Alavi, T. Deutsch, M. Bernasconi, S. Goedecker, D. Marx, M. Tuckerman and M. Parrinello, CPMD version 3.5 (2000), copyright: MPI Solid State Research Institute in Stuttgart and the IBM Research Laboratory, Zurich;

24. see for example: D. K. Remler and P. A. Madden, Mol. Phys. 70, 921 (1985). M. Sprik, J. Hutter and M. Parrinello, J. Chem. Phys. 105, 1142 (1996).

25. S. J. Weiner, P. A. Kollman, D. A. Case, U. C. Singh, C. Ghio, G. Alagona, S. Profeta and P. Weiner, J. Am. Chem. Soc. 106, 765 (1984); W. F. van Guensteren and H. J. C. Berendsen. Gromos Manual, BIOMOS, Biomolecular Software, Laboratory of Physical Chemistry, University of Groeningen, The Netherlands (1988).

26. H. J. C. Berendsen, J. P. M. Postma, W. F. van Gunsteren and J. Hermans, in Interaction Models for Water in Relation to Protein Hydratation (Reidel, Dordrecht, 1981).

27. N. T. Skipper and G. W. Neilson, J. Phys.: Condens. Matter 1, 4141 (1989).

28. L. Turi and D. Borgis, J. Chem. Phys. 117, 6186 (2002).

29. R. N. Barnett, U. Landman and C. L. Cleveland, J. Chem. Phys. 88, 4421 (1988); R. N. Barnett, U. Landman and C. L. Cleveland, J. Chem. Phys. 88, 4429 (1988); R. N. Barnett, U. Landman and A. Nitzan, J. Chem. Phys. 90, 4413 (1989).

30. P. Durand and J. C. Barthelat, Theoret. Chim. Acta 38, 283 (1975).

31. J. Hellmann, Einführung in die Quantenchemie (Deuticke, Leipzig, 1937); R. P. Feynman, Phys. Rev. 56, 340 (1939).

32. M. P. Allen and D. J. Tildesley, Computer Simulation of Liquids (Oxford University Press, Oxford, 1987).

33. S. Nosé, J. Chem. Phys. 81, 511 (1984); W. G. Hoover, Phys. Rev. A 31, 1695 (1985). 
34. C. Gear, Numerical Initial Value Problems, in Ordinary Differential Equations, Englewood Cliffs, NJ, USA (1971).

35. D. Borgis and A. Staib, J. Chem. Phys. 104, 4776 (1996).

36. M. D. Winn and D. E. Logan, J. Phys. Condens. Matter 5, 3103 (1993).

37. H. Fröhlich, Theory of Dielectrics (Oxford University, Oxford, 1958).

38. D. Laria and R. Kapral, J. Chem. Phys. 16, 7712 (2002).

39. H. Gelabert and Y. Gaudel, J. Phys. Chem. 100, 13993 (1996).

40. P. Mulvaney and A. Henglein, Chem. Phys. Lett. 168, 391 (1990).

41. J. C. Pickering and V. Zilio, Eur. Phys. J. D 13, 181 (2001).

42. H. J. Kim and J. T. Hynes, J. Phys. Chem. 94, 2736 (1990); J. Chem. Phys. 93, 5194 (1990); J. Chem. Phys. 93, 5211 (1990); J. Chem. Phys. 96, 5088 (1990).

43. J. Bonin, I. Lampre, B. Soroushian and M. Mostafavi, J. Phys. Chem. A 108, 6871 (2004). 